Copyright by Siam Publications. Russell, D. L.; White, L. W., "An elementary nonlinear beam theory with finite buckling

deformation properties," SIAM J. Appl. Math., 62(4), 1394-1413, (2002). DOI: 10.1137/050634268

\title{
AN ELEMENTARY NONLINEAR BEAM THEORY WITH FINITE BUCKLING DEFORMATION PROPERTIES*
}

\author{
DAVID L. RUSSELL ${ }^{\dagger}$ AND LUTHER W. WHITE $\ddagger$
}

\begin{abstract}
A simple nonlinear beam model is derived from basic principles. The assumption upon which the derivation is based is that axial motions are of second order compared with transverse motions of the beam. The existence of solutions is established. Issues concerning the uniqueness and nonuniqueness of solutions are examined with regard to buckling behavior. The numerical treatment of problems with nonunique solutions is presented. The results of buckling calculations are presented.
\end{abstract}

Key words. elastic beam, nonlinear beam, buckling

AMS subject classifications. 74G10, 74G15, 74G60, 74G65

PII. S0036139996309138

1. Introduction. The objectives of this work are to (i) present in one place a physical derivation of a simple extension of the classic linear beam equation to a model which can capture nonlinear effects such as buckling, (ii) derive an associated variational principle that submits itself to analysis for existence, uniqueness, and nonuniquenss, and (iii) describe some of the numerical results in which nontrivial solutions are obtained. The study of elastic structures such as beams and plates is, to a large extent, dominated by linear models. Even the most familiar model used to predict the onset of buckling in elastic beams subject to longitudinal forces is a linear one. Nevertheless, it is well understood that incorporation of both "pre-buckling" and "post-buckling" phases into a single model requires the proposed model, at the least, to account for nonlinear geometric effects.

There are numerous models of this sort in the literature, of course, such as [2], $[3],[4],[5],[6],[7],[8],[10],[11]$, and [13]. In this respect, our primary "point of departure" is to assume that "in line" or "in plane" displacements are of the same order as the square of the transverse and/or shearing displacements generally admitted into linear models of the same structures. The development of these models has been given new emphasis by recent developments in "formation theory," the deliberate modification of the configuration of an elastic body by means of attached or imbedded actuators [14]. We will not pursue such a study in this paper, leaving that work for another article. The objective here is to present a derivation of the model from first principles and to indicate how the model may be useful for the analysis of "buckling"-type bifurcation problems, not only with regard to prediction of "critical" loads initiating such bifurcation, already possible with the standard linear model, but also permitting studies of the progressive growth of the buckling phenomenon as the load is increased beyond the critical buckling load.

In section 2, we derive a set of geometrically nonlinear equations for the elastic beam. These equations compare with those presented in [10]. Here, however, derivations, although ad hoc, are based on explicitly stated fundamental principles. Then, in section 3 , we demonstrate existence and uniqueness of solutions from a variational

\footnotetext{
* Received by the editors September 13, 1996; accepted for publication (in revised form) October 10, 2001; published electronically April 19, 2002.

http://www.siam.org/journals/siap/62-4/30913.html

$\dagger$ Department of Mathematics, Virginia Tech, Blacksburg, VA 24061-0123 (russell@math.vt.edu).

${ }^{\ddagger}$ Department of Mathematics, The University of Oklahoma, Norman, OK 73019 (Lwhite@math. ou.edu).
} 


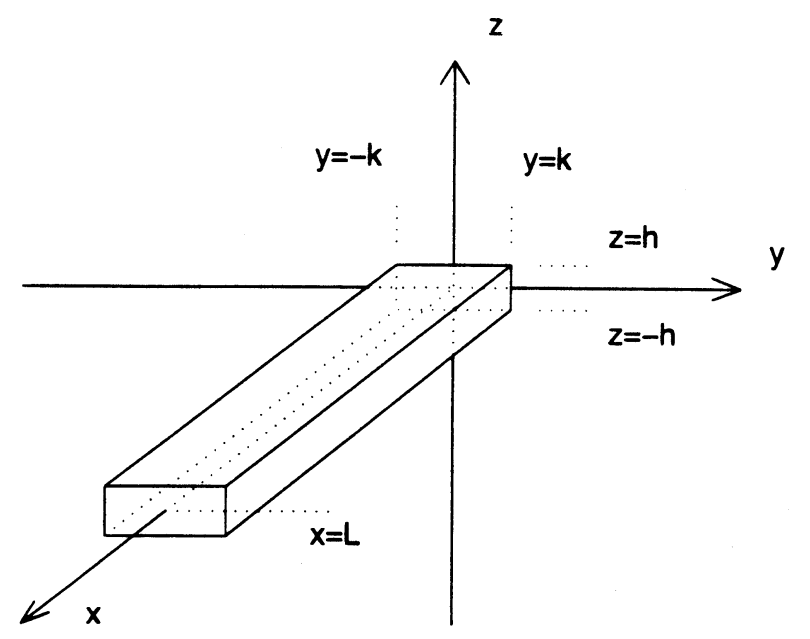

FIG. 2.1. Beam configuration.

standpoint. Questions of uniqueness of equilibria particularly related to buckling phenomena are further developed in sections 4 and 5. Section 6 is devoted to reporting the results of several numerical experiments.

2. Geometrically nonlinear beam equations. Let us consider an elastic beam of length $L$, with uniform cross section, occupying the region $0 \leq x \leq L,-k \leq$ $y \leq k,-h \leq z \leq h$ in $\mathbf{R}^{3}$, as shown in Figure 2.1.

In equilibrium the elastic axis coincides with the $x$-axis. The displaced elastic axis, or "neutral curve," admitting both transverse or lateral and in line displacements, is described by

$$
\left[\begin{array}{l}
x_{d} \\
y_{d}
\end{array}\right]=\left[\begin{array}{c}
x+\xi(x) \\
\eta(x)
\end{array}\right]
$$

where $\xi(x)$ and $\eta(x)$ are (at least) piecewise $\mathbf{C}^{1}$ and $\mathbf{C}^{2}$ functions, where $x \in(0, L)$. Such a deformation results in an infinitessimal stretching or contraction of the neutral curve in the form, with ' denoting the derivative with respect to $x$,

$$
d s=\left[\left(1+\xi^{\prime}\right)^{2}+\left(\eta^{\prime}\right)^{2}\right]^{1 / 2} d x .
$$

If $E$ is Young's modulus, the potential energy due to the stretching of the neutral curve is given by

$$
V_{N}=\frac{1}{2} E A \int_{0}^{L}\left[\left[\left(1+\xi^{\prime}\right)^{2}+\left(\eta^{\prime}\right)^{2}\right]^{1 / 2}-1\right]^{2} d x .
$$

Our basic assumption, described in section 1 , is that $\xi$ and $\xi^{\prime}$ are of the same order as $\eta^{2}$ and $\eta^{2}$, respectively, the higher powers in each case being of a lower order of magnitude. This assumption allows us to begin our analysis by developing an expression for the potential energy due to compression/dilation parallel to the elastic axis. The cross-sectional area being

$$
A=4 k h,
$$




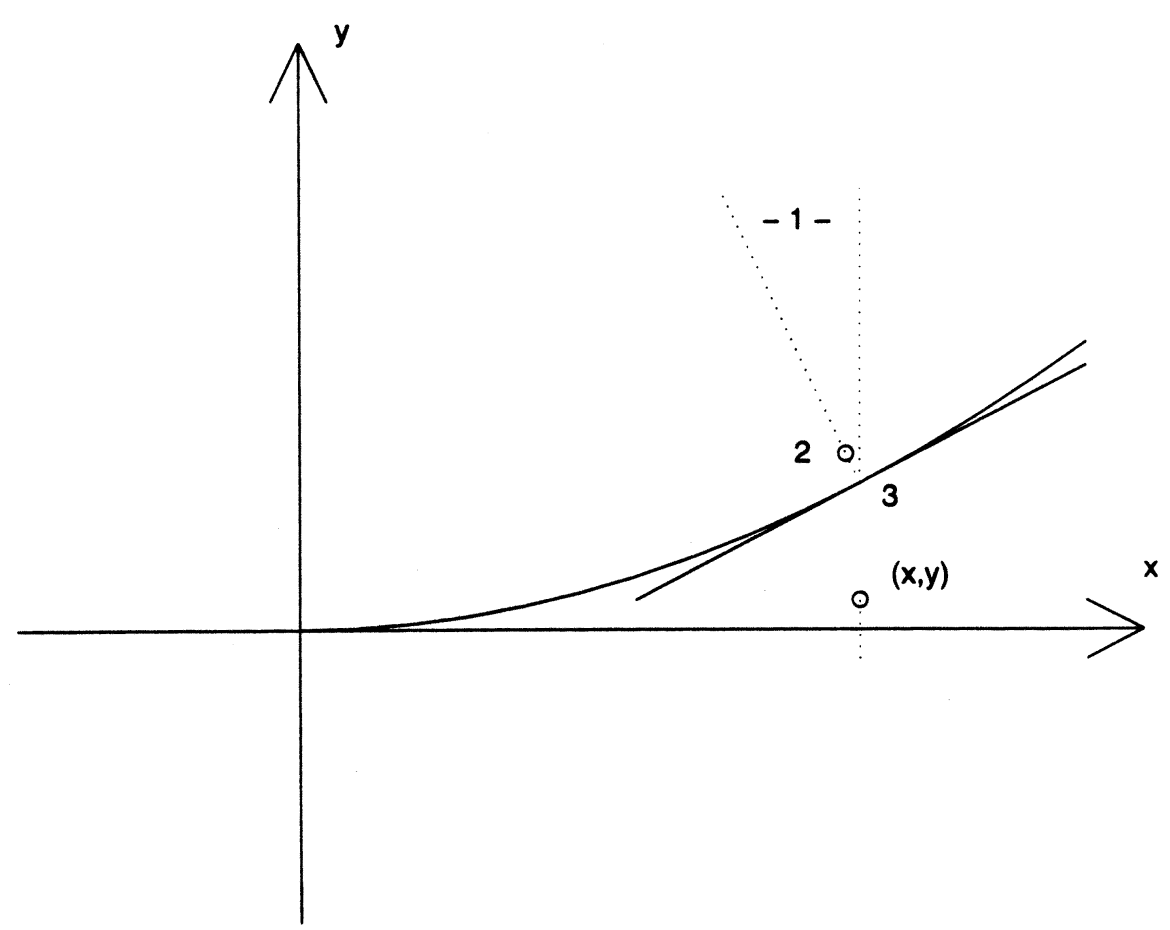

FiG. 2.2. Displaced coordinates. (1) $\psi(x)$, (2) $(x+\xi(x)-y \sin (\psi(x)), \eta(x)-y \cos (\psi(x)))$, (3) $(x+\xi(x), \eta(x))$.

that energy can be expressed in terms of the length differential:

$$
\begin{gathered}
V_{N}=\frac{1}{2} E A \int_{0}^{L}\left\{\left[\left(1+\xi^{\prime}\right)^{2}+\left(\eta^{\prime}\right)^{2}\right]^{1 / 2}-1\right\}^{2} d x \\
\simeq \text { (with our assumptions) } \\
\frac{1}{2} E A \int_{2}^{L}\left\{\left[1+\xi^{\prime}+\frac{\left(\eta^{\prime}\right)^{2}}{2}\right]-1\right\}^{2} d x \\
\simeq \frac{E k h}{2} \int_{0}^{L}\left[2 \xi^{\prime}+\left(\eta^{\prime}\right)^{2}\right]^{2} d x
\end{gathered}
$$

Henceforth, we identify $V_{N}$ with the last approximation here.

Points not on the elastic axis in equilibrium, i.e., those with $y \neq 0$, are assumed to have displaced coordinates, as shown in Figure 2.2, given by

$$
\left[\begin{array}{l}
\hat{x} \\
\hat{y}
\end{array}\right]=G(x, y)=\left[\begin{array}{c}
x+\xi(x)-y \sin (\psi(x)) \\
\eta(x)-y \cos (\psi(x))
\end{array}\right],
$$

where $\psi(x)$ is the rotation angle at $x \in(0, L)$. We assume that the angle $\psi(x)$ is small enough so that the approximations $\psi(x) \approx \tan \psi(x) \approx \sin \psi(x)$ suffice for our 
analysis. Thus, with our assumptions, we may identify $\psi(x)$ with the slope $\eta^{\prime}(x)$. The deformation functions $u_{1}$ and $u_{2}$ are then

$$
\left[\begin{array}{l}
u_{1}(x, y) \\
u_{2}(x, y)
\end{array}\right]=G(x, y)-\left[\begin{array}{l}
x \\
y
\end{array}\right]=\left[\begin{array}{c}
\xi(x)-y \sin (\psi(x)) \\
\eta(x)-y(1-\cos (\psi(x)))
\end{array}\right] .
$$

Since $1-\cos \psi=O\left(\psi^{2}\right)=O\left(\left(\eta^{\prime}\right)^{2}\right),(2.5)$ reduces, with our assumptions, to

$$
\left[\begin{array}{l}
u_{1}(x, y) \\
u_{2}(x, y)
\end{array}\right]=\left[\begin{array}{c}
\xi(x)-y \eta^{\prime}(x) \\
\eta(x)
\end{array}\right] .
$$

Defining the strains in the manner standard for linear elasticity [9], these are

$$
\begin{aligned}
& \epsilon_{11}=\frac{\partial u_{1}}{\partial x}=\xi^{\prime}-y \eta^{\prime \prime}, \quad \epsilon_{22}=\frac{\partial u_{2}}{\partial y}=0, \\
& \epsilon_{12}=\frac{1}{2}\left[\frac{\partial u_{2}}{\partial x}+\frac{\partial u_{1}}{\partial y}\right]=\frac{1}{2}\left(\eta^{\prime}-\eta^{\prime}\right)=0 .
\end{aligned}
$$

The corresponding stresses are

$$
\begin{gathered}
\sigma_{11}=\frac{E}{1-\mu^{2}}\left[\epsilon_{11}+\mu \epsilon_{22}\right]=\frac{E}{1-\mu^{2}} \epsilon_{11}=\frac{E}{1-\mu^{2}}\left(\xi^{\prime}-y \eta^{\prime \prime}\right) \\
\sigma_{22}=\frac{E}{1-\mu^{2}}\left[\epsilon_{22}+\mu \epsilon_{11}\right]=\frac{E \mu}{1-\mu^{2}} \epsilon_{11} .
\end{gathered}
$$

Because $\epsilon_{12}=\epsilon_{22}=0$, the potential energy due to bending may then be expressed as

$$
\begin{aligned}
V_{B} & =\frac{1}{2} \int_{0}^{L} \int_{-k}^{k} \int_{-h}^{h} \epsilon_{11} \sigma_{11} d x d y d x \\
& =\frac{1}{2} \int_{0}^{L} \int_{-k}^{k} \int_{-h}^{h} \frac{E}{1-\mu^{2}}\left[\xi^{\prime}-y \eta^{\prime \prime}\right]^{2} d z d y d x \\
& =\frac{E}{2\left(1-\mu^{2}\right)} \int_{0}^{L} \int_{-k}^{k} \int_{-h}^{h}\left[\left(\xi^{\prime}\right)^{2}-2 y \xi^{\prime} \eta^{\prime \prime}+y^{2}\left(\eta^{\prime \prime}\right)^{2}\right] d z d y d x \\
& =\frac{2 h E}{\left(1-\mu^{2}\right)} \int_{0}^{L}\left[k\left(\xi^{\prime}\right)^{2}+\frac{k^{3}}{3}\left(\eta^{\prime \prime}\right)^{2}\right] d x .
\end{aligned}
$$

Assuming an applied lateral force $f(x)$, a longitudinal force $g_{l}$ applied at $x=L$, a distributed longitudinal force $g(x)$, and a torque $g_{\tau}$ applied at $x=L$, we may write the total potential energy as

$$
\begin{aligned}
V(\xi, \eta) & =\frac{E k h}{2} \int_{0}^{L}\left[2 \xi^{\prime}+\left(\eta^{\prime}\right)^{2}\right]^{2} d x+\frac{2 h E}{1-\mu^{2}} \int_{0}^{L}\left[k\left(\xi^{\prime}\right)^{2}+\frac{k^{3}}{3}\left(\eta^{\prime \prime}\right)^{2}\right] d x \\
& -\int_{0}^{L}[f(x) \eta(x)+g(x) \xi(x)] d x-g_{l} \xi(L)-g_{\tau} \eta^{\prime}(L) .
\end{aligned}
$$


As a first step toward obtaining the equilibrium equations, we compute the first order variation of $\mathrm{V}$ with respect to small displacements in $\xi$ and $\eta$ :

$$
\begin{gathered}
\delta V(\xi, \eta)(\delta \xi, \delta \eta)=E k h \int_{0}^{L}\left(2 \xi^{\prime}+\left(\eta^{\prime}\right)^{2}\right)\left(2 \delta \xi^{\prime}+2 \eta^{\prime} \delta \eta^{\prime}\right) d x-\int_{0}^{L}(f \delta \eta+g \delta \xi) d x \\
+\frac{2 h E}{1-\mu^{2}} \int_{0}^{L}\left[2 k \xi^{\prime} \delta \xi^{\prime}+\frac{2 k^{3}}{3} \eta^{\prime \prime} \delta \eta^{\prime \prime}\right] d x-g_{l} \delta \xi(L)-g_{\tau} \delta \eta^{\prime}(L) .
\end{gathered}
$$

Integrating by parts and setting the resulting expression equal to zero, this becomes

$$
\begin{gathered}
2 E k h\left\{\left[\left(2 \xi^{\prime}+\left(\eta^{\prime}\right)^{2}\right)\left(\delta \xi+\eta^{\prime} \delta \eta\right)\right]_{0}^{L}-\int_{0}^{L}\left[\left(2 \xi^{\prime}+\left(\eta^{\prime}\right)^{2}\right)^{\prime} \delta \xi+\left(\left(2 \xi^{\prime}+\left(\eta^{\prime}\right)^{2}\right) \eta^{\prime}\right)^{\prime} \delta \eta\right] d x\right\} \\
(2.11)+\frac{4 h E}{1-\mu^{2}}\left\{\left[k \xi^{\prime} \delta \xi+\frac{k^{3}}{3} \eta^{\prime \prime} \delta \eta^{\prime}-\frac{k^{3}}{3} \eta^{\prime \prime \prime} \delta \eta\right]_{0}^{L}-\int_{0}^{L}\left[k \xi^{\prime \prime} \delta \xi-\frac{k^{3}}{3} \eta^{\prime \prime \prime \prime} \delta \eta\right] d x\right\} \\
-\int_{0}^{L}[f \delta \eta+g \delta \xi] d x-g_{l} \delta \xi(L)-g_{\tau} \delta \eta^{\prime}(L)=0 .
\end{gathered}
$$

As always in problems of this type, the boundary conditions depend on the physical situation at each endpoint. For definiteness here, and because it corresponds to the example we wish to study at some length later in the paper, we will assume the beam to be in the cantilevered configuration. (We also study the case in which the boundary is pinned at $x=L$, though less extensively, in sections 5 and 6 .) Thus, at $x=0$, we assume the beam is clamped:

$$
\xi(0)=0, \quad \eta(0)=0, \quad \eta^{\prime}(0)=0 .
$$

At $x=L$, the beam is free, and we set the coefficients of the boundary variations equal to zero in order to obtain the boundary conditions:

$$
\begin{gathered}
2 E k h\left\{\left[2 \xi^{\prime}(L)+\eta^{\prime}(L)^{2}\right]+\frac{2 \xi^{\prime}(L)}{1-\mu^{2}}\right\}=g_{l}, \\
{\left[\left(2 \xi^{\prime}(L)+\eta^{\prime}(L)^{2}\right) \eta^{\prime}(L)\right]-\frac{2 k^{2}}{3\left(1-\mu^{2}\right)} \eta^{\prime \prime \prime}(L)=0,} \\
\frac{4 E k^{3} h}{\left(1-\mu^{2}\right)} \eta^{\prime \prime}(L)=g_{\tau} .
\end{gathered}
$$

Setting the coefficients of $\delta \xi$ and $\delta \eta$ in the integral terms of (2.11) equal to zero, we obtain the equations

$$
\begin{gathered}
\frac{4 E k h}{1-\mu^{2}} \xi^{\prime \prime}+2 E k h\left(2 \xi^{\prime}+\left(\eta^{\prime}\right)^{2}\right)^{\prime}+g=0, \\
\frac{4 E k^{3} h}{3\left(1-\mu^{2}\right)} \eta^{\prime \prime \prime \prime}-2 E k h\left(\left(2 \xi^{\prime}+\left(\eta^{\prime}\right)^{2}\right) \eta^{\prime}\right)^{\prime}=f .
\end{gathered}
$$


It will be convenient to redefine $g_{l}, g_{\tau}, f$, and $g$ as those same quantities divided by $4 E k h$. Then with

$$
a_{1}=\frac{1}{1-\mu^{2}}, \quad a_{2}=\frac{2 k^{2}}{3\left(1-\mu^{2}\right)},
$$

we have the boundary conditions

$$
\begin{gathered}
\left(1+a_{1}\right) \xi^{\prime}(L)+\frac{\eta^{\prime}(L)^{2}}{2}=g_{l}, \\
\left(\xi^{\prime}(L)+\frac{\eta^{\prime}(L)^{2}}{2}\right) \eta^{\prime}(L)-a_{2} \eta^{\prime \prime \prime}(L)=0, \\
a_{2} \eta^{\prime \prime}(L)=g_{\tau},
\end{gathered}
$$

together with the equations

$$
\begin{aligned}
& \left(1+a_{1}\right) \xi^{\prime \prime}+\left[\frac{\left(\eta^{\prime}\right)^{2}}{2}\right]^{\prime}+g=0 \\
& a_{2} \eta^{\prime \prime \prime \prime}-\left[\left(\xi^{\prime}+\frac{\left(\eta^{\prime}\right)^{2}}{2}\right) \eta^{\prime}\right]^{\prime}=f .
\end{aligned}
$$

3. Existence and uniqueness of solutions. We consider (2.19)-(2.23) developed in section 2. We begin our study of these equations by defining the spaces

$$
\begin{gathered}
V_{1}=\left\{\phi \in H^{1}(0, L): \phi(0)=0\right\}, \\
V_{2}=\left\{\psi \in H^{2}(0, L): \psi(0)=\psi^{\prime}(0)=0\right\} .
\end{gathered}
$$

It should be noted that there are positive numbers $\gamma_{0}$ and $\gamma_{1}$ such that, for $\phi \in V_{1}$ and $\psi \in V_{2}$, we have [1]

$$
\gamma_{0}\left\|\phi^{\prime}\right\| \geq\|\phi\|, \quad \gamma_{1}\left\|\psi^{\prime \prime}\right\| \geq\|\psi\|,
$$

where $\|\cdot\|$ denotes the usual norm for $L^{2}(0, L)$.

Using (2.22) and the boundary condition (2.19), we observe that, for $\phi \in V_{1}$, we have

$$
\begin{aligned}
0= & \int_{0}^{L}\left\{\left(1+a_{1}\right) \xi^{\prime}+\frac{\left(\eta^{\prime}\right)^{2}}{2}\right\} \phi d x \\
=\left\{\left(1+a_{1}\right) \xi^{\prime}(L)+\frac{\eta^{\prime}(L)^{2}}{2}\right\} \phi(L) & -\int_{0}^{L}\{\phi d x \\
& +\int_{0}^{L} g \phi d x \\
= & g_{l} \phi(L)-\int_{0}^{L}\left\{\left(1+a_{1}\right) \xi^{\prime}+\frac{\left(\eta^{\prime}\right)^{2}}{2}\right\} \phi^{\prime} d x \\
&
\end{aligned}
$$


Then, using (2.23) and the boundary condition (2.20), we further have, for all $\psi \in V_{2}$,

$$
\begin{aligned}
0= & -\int_{0}^{L}\left\{a_{2} \eta_{2}^{\prime \prime \prime}-\left(\left(\xi^{\prime}+\frac{\left(\eta^{\prime}\right)^{2}}{2}\right) \eta^{\prime}\right)\right\}^{\prime} \psi d x+\int_{0}^{L} f \psi d x \\
= & \int_{0}^{L}\left\{a_{2} \eta^{\prime \prime \prime}-\left(\left(\xi^{\prime}+\frac{\left(\eta^{\prime}\right)^{2}}{2}\right) \psi^{\prime} d x+\int_{0}^{L} f \psi d x\right.\right. \\
= & \text { (integrating the } \left.\eta^{\prime \prime \prime} \text { term by parts again and using }(2.21)\right) \\
& \frac{a_{2}}{k^{2}} g_{\tau} \psi^{\prime}(L)-\int_{0}^{L}\left\{a_{2} \eta^{\prime \prime} \psi^{\prime \prime}+\left(\left(\xi^{\prime}+\frac{\left(\eta^{\prime}\right)^{2}}{2}\right) \eta^{\prime}\right) \psi^{\prime}\right\} d x+\int_{0}^{L} f \psi d x
\end{aligned}
$$

We introduce the functional

$$
\begin{gathered}
J(\xi, \eta)=\int_{0}^{L}\left\{\frac{1}{2}\left\{a_{1}\left(\xi^{\prime}\right)^{2}+a_{2}\left(\eta^{\prime \prime}\right)^{2}+\left(\xi^{\prime}+\frac{\left(\eta^{\prime}\right)^{2}}{2}\right)^{2}\right\}-g \xi-f \eta\right\} d x \\
-\frac{a_{2}}{k^{2}} g_{\tau} \eta^{\prime}(L)-g_{l} \xi(L),
\end{gathered}
$$

that is, the total potential energy functional (2.10). We compute the differential and note that the variations $\delta \xi$ and $\delta \eta$ satisfy the conditions imposed on $\phi$ and $\eta$ in the definitions of $V_{1}$ and $V_{2}$, respectively. Hence we observe the following.

LEMma 3.1. The satisfaction of (3.5) and (3.6) determines precisely the set of first order necessary conditions for the minimization of $J(\xi, \eta)$ over $V_{1} \times V_{2}$.

To prove the existence of a solution to the boundary value problem (3.4)-(3.5), we prove that there exists a minimizer of $J(\cdot, \cdot)$ over $V_{1} \times V_{2}$. Now, with $V_{1}$ and $V_{2}$ as in (3.1) and (3.2), let

$$
\mathcal{V}=V_{1} \times V_{2}, \quad \omega=(\xi, \eta), \quad J(\omega)=J(\xi, \eta) .
$$

THEOREM 3.2. There exists a solution to the following minimization problem. Find $\omega_{0} \in \mathcal{V}$ such that

$$
J\left(\omega_{0}\right)=\operatorname{infimum}\{J(\omega): \omega \in \mathcal{V}\} .
$$

Proof. We first show that indeed $J(\omega)$ is bounded below. Observe that

$$
J(\omega) \geq \int_{0}^{L}\left\{\frac{a_{1}}{2}\left(\xi^{\prime}\right)^{2}-g \xi+\frac{a_{2}}{2}\left(\eta^{\prime \prime}\right)^{2}-f \eta\right\} d x-\frac{a_{2}}{k^{2}} g_{\tau} \eta^{\prime}(L)-g_{l} \xi(L) .
$$

Since the mappings $\xi \mapsto \xi(L)$ and $\eta \mapsto \eta^{\prime}(L)$ are continuous linear functionals on $V_{1}$ and $V_{2}$, respectively, we have, for some $b_{1}$ and $b_{2}$,

$$
J(\omega) \geq \frac{a_{1}}{2}\left\|\xi^{\prime}\right\|^{2}-\left(\|g\|+b_{1}\right)\|\xi\|+\frac{a_{2}}{2}\left\|\eta^{\prime \prime}\right\|^{2}-\left(\|f\|+b_{2}\right)\|\eta\| .
$$

Using the inequalities (3.3), we see that there are positive numbers $\alpha$ and $\beta$ such that

$$
J(\omega) \geq \alpha\|\omega\|_{\mathcal{V}}^{2}-\beta\|\omega\|_{\mathcal{V}} .
$$

Then it is easy to see that $J(\omega)$ is bounded below, specifically,

$$
J(\omega) \geq-\frac{\beta^{2}}{4 \alpha}, \quad \omega \in \mathcal{V} .
$$


To demonstrate the existence of a minimum, let

$$
d=\operatorname{infimum}\{J(\omega): \omega \in \mathcal{V}\},
$$

and let $\left\{\omega_{n}\right\}_{n=1}^{\infty} \subset \mathcal{V}$ be a sequence such that $J\left(\omega_{n}\right) \rightarrow d$ as $n \rightarrow \infty$. Let $N$ be such that

$$
d \leq J\left(\omega_{n}\right) \leq d+1, \quad n \geq N
$$

Then, from (3.7),

$$
d+1 \geq \alpha\left\|\omega_{n}\right\|_{\mathcal{V}}^{2}-\beta\left\|\omega_{n}\right\|_{\mathcal{V}}
$$

from which we easily infer that

$$
0 \leq\left\|\omega_{n}\right\|_{\mathcal{V}} \leq \frac{1}{2 \alpha}\left[\beta+\left(\beta^{2}+4 \alpha(d+1)\right)^{\frac{1}{2}}\right]
$$

so that $\left\{\omega_{n}\right\}_{n=1}^{\infty}$ is a bounded sequence in $\mathcal{V}$. Thus we can find a subsequence, for which we will use the same designation, such that

$$
\omega_{n}=\left(\xi_{n}, \eta_{n}\right) \rightarrow \omega_{0}=\left(\xi_{0}, \eta_{0}\right)
$$

weakly in $\mathcal{V}=V_{1} \times V_{2}$. The weak convergence of $\left\{\xi_{n}\right\}_{n=1}^{\infty}$ to $\xi_{0}$ in $V_{1}$ and $\left\{\eta_{n}\right\}_{n=1}^{\infty}$ to $\eta_{0}$ in $V_{2}$ imply, respectively, the weak convergence of $\left\{\xi_{n}^{\prime}\right\}_{n=1}^{\infty}$ to $\xi_{0}^{\prime}$ in $L^{2}(0, L)$ and the weak convergence of $\left\{\eta_{n}^{\prime}\right\}_{n=1}^{\infty}$ to $\eta^{\prime}$ in $H^{1}(0, L)$ and hence strong convergence in $L^{4}(0, L)$. Now from the inequality in $L^{4}(0, L)$ resulting from the Schwarz inequality, we have

$$
\begin{aligned}
& \int_{0}^{L}\left[f^{2}-g^{2}\right]^{2} d x=\int_{0}^{L}[f+g]^{2}[f-g]^{2} d x \\
& \leq\left\{\int_{0}^{L}[f+g]^{4} d x\right\}^{\frac{1}{2}}\left\{\int_{0}^{L}[f-g]^{4} d x\right\}^{\frac{1}{2}} .
\end{aligned}
$$

From this it follows that $\left\{\left(\eta_{n}^{\prime}\right)^{2}\right\}_{n=1}^{\infty}$ converges to $\left(\eta_{0}^{\prime}\right)^{2}$ strongly in $L^{2}(0, L)$. Thus we see that

$$
\xi_{n}^{\prime}+\frac{\left(\eta_{n}^{\prime}\right)^{2}}{2} \rightarrow \xi_{0}^{\prime}+\frac{\left(\eta_{0}^{\prime}\right)^{2}}{2},
$$

weakly in $L^{2}(0, L)$. Since it is also clear that $\xi_{n}(L) \rightarrow \xi_{0}(L)$ and $\eta_{n}^{\prime}(L) \rightarrow \eta_{0}(L)$, we have

$$
d=\liminf J\left(\omega_{n}\right) \geq J\left(\omega_{0}\right) \geq d
$$

and $J\left(\omega_{0}\right)=d$. We conclude that $\omega_{0}$ is a solution to the minimization problem.

Since any minimizing element satisfies the necessary conditions, it follows that there is a weak solution of (2.19)-(2.23).

Corollary 3.3. There exists a solution to the boundary value problem (2.19)$(2.23)$. 
At this point, let us consider the special case arising when $g=0$ and $g_{l}=g_{\tau}=0$, i.e., when only lateral forces are present. Then we may integrate (2.22) to obtain

$$
\xi^{\prime}=-\frac{\left(\eta^{\prime}\right)^{2}}{2\left(1+a_{1}\right)}
$$

and substitute into (2.23) to obtain

$$
a_{2} \eta^{\prime \prime \prime \prime}-\left[\frac{a_{1}\left(\eta^{\prime}\right)^{3}}{2\left(1+a_{1}\right)}\right]^{\prime}=f
$$

with boundary conditions (still in the cantilevered case)

$$
\begin{gathered}
\eta(0)=\eta^{\prime}(0)=0 \\
\eta^{\prime \prime}(L)=\left[a_{2} \eta^{\prime \prime \prime}-\frac{a_{1}\left(\eta^{\prime}\right)^{3}}{2\left(1+a_{1}\right)}\right](L)=0 .
\end{gathered}
$$

For $\psi \in V_{2}$, integrating the product of $\psi$ with (3.8), then integrating by parts with the use of $\psi(0)=\psi^{\prime}(0)=0$, we obtain

$$
\int_{0}^{L}\left[a_{2} \eta^{\prime \prime} \psi^{\prime \prime}+\frac{a_{1}\left(\eta^{\prime}\right)^{3}}{2\left(1+a_{1}\right)} \psi^{\prime}\right] d x=\int_{0}^{L} f \psi d x
$$

We now introduce the potential energy functional

$$
J(\eta)=\int_{0}^{L}\left[\frac{a_{2}}{2}\left(\eta^{\prime \prime}\right)^{2}+\frac{a_{1}\left(\eta^{\prime}\right)^{4}}{8\left(1+a_{1}\right)}-f \eta\right] d x .
$$

The existence of a minimizer of the functional $J(\cdot)$ of (3.12) may be deduced from the existence previously demonstrated or deduced directly using arguments similar to those used previously. However, in the present case, we can prove uniqueness since the functional $J(\cdot)$ is easily seen to be strictly convex.

Proposition 3.4. There exists a unique solution to the following minimization problem.

Find $\eta_{0}$ such that

$$
J\left(\eta_{0}\right)=\operatorname{infimum}\left\{J(\eta): \eta \in V_{2}\right\}
$$

COROllary 3.5. There is a unique solution to the variational problem (3.11) and thus to (3.8)-(3.10).

Remark 3.1. It should be noted that the term

$$
\left[\xi^{\prime}+\frac{\left(\eta^{\prime}\right)^{2}}{2}\right]^{2}
$$

in (3.6) is not convex so that $J(\xi, \eta)$ is not a convex functional in general, suggesting that for $g \neq 0$ or $g_{l} \neq 0$ there may exist multiple solutions such as one would anticipate, for example, in the case of buckling in response to a longitudinally applied load. We will discuss this in more detail in the next section. 
4. Uniqueness and nonuniqueness of equilibrium states in the presence of longitudinal loading. Let us define

$$
-l(x)=g_{l}+\int_{x}^{L} g(s) d s .
$$

Then $l^{\prime}(x)=g(x)$, and we have, for $\xi \in V_{1}$,

$$
\begin{aligned}
\int_{0}^{L} g(x) \xi(x) d x & =\int_{0}^{L} l^{\prime}(x) \xi(x) d x=\left.l(x) \xi(x)\right|_{0} ^{L}-\int_{0}^{L} l(x) \xi^{\prime}(x) d x \\
& =-g_{l} \xi(L)-\int_{0}^{L} l(x) \xi^{\prime}(x) d x .
\end{aligned}
$$

Integrating (2.22) from $x$ to $L$ and using (2.19), we see that

$$
\left(1+a_{1}\right) \xi^{\prime}(x)+\frac{\eta^{\prime}(x)^{2}}{2} \equiv-l(x), \quad x \in(0, L),
$$

and thus

$$
\xi^{\prime}(x)=-\frac{l(x)+\eta^{\prime}(x)^{2} / 2}{1+a_{1}}
$$

so that

$$
a_{2} \eta^{\prime \prime \prime \prime}-\left[\frac{-l+a_{1}\left(\eta^{\prime}\right)^{2} / 2}{1+a_{1}} \eta^{\prime}\right]^{\prime}=f
$$

Assuming $g_{\tau}=0$, the boundary conditions (2.12) and (2.19)-(2.21) are now replaced by

$$
\eta(0)=\eta^{\prime}(0)=\eta^{\prime \prime}(L)=0
$$

and

$$
a_{2} \eta^{\prime \prime \prime}(L)-\frac{g_{l}+a_{1} \eta^{\prime}(L)^{2} / 2}{1+a_{1}} \eta^{\prime}(L)=0 .
$$

It is easy to see that (4.4) and the boundary conditions (4.5) and (4.6) may be given the weak formulation in $V_{2}$ :

$$
\int_{0}^{L}\left\{a_{2} \eta^{\prime \prime}(x) \phi^{\prime \prime}(x)+\frac{-l(x)+\eta^{\prime}(x)^{2} / 2}{1+a_{1}} \eta^{\prime}(x) \phi^{\prime}(x)-f(x) \phi(x)\right\} d x=0
$$

for any $\phi \in V_{2}$. We consider the special case $f \equiv 0$ and introduce the functional

$$
\mathbf{J}(\eta)=\int_{0}^{L}\left\{\frac{1}{2} a_{2} \eta^{\prime \prime}(x)^{2}+\frac{-l(x) \eta^{\prime}(x)^{2} / 2+a_{1} \eta^{\prime}(x)^{4} / 8}{1+a_{1}}\right\} d x .
$$

It is clear that $\eta(x) \equiv 0$ is a solution of (4.7) and thus is a stationary point for (4.8). We next determine conditions under which there are nontrivial stationary points of $\mathbf{J}(\cdot)$. 
LEMma 4.1. If

$$
\int_{0}^{L} x^{2} l(x) d x=L a_{2}\left(1+a_{1}\right)+K_{0}
$$

for some $K_{0}>0$, then there are elements $\eta$ in $\mathbf{V}$ for which $\mathbf{J}(\eta)<0$.

Proof. Consider functions of the form

$$
\eta_{\alpha}(x)=\alpha x^{2} \in \mathbf{V}
$$

for $\alpha \in \mathbf{R}$. A short computation shows that

$$
\mathbf{J}\left(\eta_{\alpha}\right)=\left[2 a_{2} L-\frac{2}{1+a_{1}} \int_{0}^{L} x^{2} l(x) d x\right] \alpha^{2}+2 a_{1} \frac{L^{5}}{5\left(1+a_{1}\right)} \alpha^{4} .
$$

If $l(x)$ is chosen, via $g(x)$ and $g_{l}$, so that

$$
\int_{0}^{L} x^{2} l(x) d x=L a_{2}\left(1+a_{1}\right)+K_{0}, \quad K_{0}>0
$$

(it should be noted that, for a given positive $K_{0}$, this can be done, for example, by taking $g_{l}$ to be a sufficiently large negative number), then we see easily that $\mathbf{J}\left(\eta_{\alpha}\right)<0$ for small nonzero values of $\alpha$. Hence $\eta \equiv 0$ does not minimize $\mathbf{J}(\eta)$.

That a nonzero minimum must exist is demonstrated in the following theorem.

THEOREM 4.2. For all piecewise continuous longitudinal "loading functions" $l(x)$, there exists a minimizing $\eta_{0} \in V_{2}$ for $\mathbf{J}(\eta)$.

Proof. We first show that $\mathbf{J}(\eta)$ is bounded below. Suppose $\gamma_{0}$ is such that, for $\eta \in V_{2}$,

$$
\gamma_{0}^{2} \int_{0}^{L}\left(\eta^{\prime \prime}(x)\right)^{2} d x \geq \int_{0}^{L}\left(\eta^{\prime}(x)\right)^{2} d x
$$

such an inequality is easily obtained by integration. By the Schwarz inequality,

$$
\left[\int_{0}^{L}\left(\eta^{\prime}(x)\right)^{2} d x\right]^{2} \leq L \int_{0}^{L}\left(\eta^{\prime}(x)\right)^{4} d x
$$

Thus (cf. (4.8))

$$
\begin{aligned}
\mathbf{J}(\eta) & =\int_{0}^{L}\left\{\frac{1}{2} a_{2} \eta^{\prime \prime}(x)^{2}+\frac{1}{1+a_{1}}\left[-l(x) \eta^{\prime}(x)^{2} / 2+a_{1} \eta^{\prime}(x)^{4} / 8\right]\right\} d x \\
& \geq \int_{0}^{L}\left\{\left[\frac{a_{2}}{2 \gamma_{0}^{2}}-\frac{l(x)}{2\left(1+a_{1}\right)}\right] \eta^{\prime}(x)^{2}+\frac{a_{1}}{8\left(1+a_{1}\right)} \eta^{\prime}(x)^{4}\right\} d x \\
& \geq \int_{0}^{L}\left[\frac{a_{2}}{2 \gamma_{0}^{2}}-\frac{l(x)}{2\left(1+a_{1}\right)}\right] \eta^{\prime}(x)^{2} d x+\frac{a_{1}}{8 L\left(1+a_{1}\right)}\left[\int_{0}^{L} \eta^{\prime}(x)^{2} d x\right]^{2} .
\end{aligned}
$$

Since $l(x)$ is piecewise continuous on $(0, L)$, we may suppose that $M_{l}>0$ is chosen large enough so that

$$
M_{l} \int_{0}^{L} \phi(x)^{2} d x \geq \int_{0}^{L} l(x) \phi(x)^{2} d x
$$


for any $\phi \in L^{2}(0, L)$ and

$$
\frac{M_{l}}{1+a_{1}}>\frac{a_{2}}{2 \gamma_{0}^{2}}
$$

Then

$$
\mathbf{J}(\eta) \geq\left[\frac{a_{2}}{2 \gamma_{0}^{2}}-\frac{M_{l}}{1+a_{1}}\right] \int_{0}^{L} \eta^{\prime}(x)^{2} d x+\frac{a_{1}}{8 L\left(1+a_{1}\right)}\left[\int_{0}^{L} \eta^{\prime}(x)^{2} d x\right]^{2}
$$

Let us set

$$
\begin{gathered}
K_{0}=-\left\{\frac{a_{2}}{2 \gamma_{0}^{2}}-\frac{M_{l}}{1+a_{1}}\right\}, \\
K_{1}=\frac{a_{1}}{8 L\left(1+a_{1}\right)},
\end{gathered}
$$

and

$$
\beta=\int_{0}^{L} \eta^{\prime}(x)^{2} d x
$$

Thus inequality (4.11) yields

$$
\mathbf{J}(\eta) \geq-K_{0} \beta+K_{1} \beta^{2} .
$$

The minimum of the right side of (4.12) occurs for $\beta=\frac{K_{0}}{2 K_{1}}$. The computed minimum value there together with (4.12) then provides the lower bound

$$
\mathbf{J}(\eta) \geq \frac{-K_{0}^{2}}{4 K_{1}}
$$

for any $\eta \in V_{2}$. Thus we may define

$$
d=\inf \left\{\mathbf{J}(\eta): \eta \in V_{2}\right\} .
$$

Let $M>d$ and $V_{M}$ denote the set of $\eta \in V_{2}$ such that $\mathbf{J}(\eta) \leq M$. We will show that there is another positive constant $M_{1}$ such that $\left\|\eta^{\prime \prime}\right\| \leq M_{1}$. We have

$$
\begin{gathered}
\mathbf{J}(\eta)=\int_{0}^{L}\left\{\frac{1}{2} a_{2} \eta^{\prime \prime}(x)^{2}+\frac{1}{1+a_{1}}\left[-l(x)\left(\eta^{\prime}(x)^{2} / 2\right)+a_{1}\left(\eta^{\prime}(x)^{4} / 8\right)\right]\right\} d x \\
\geq \frac{a_{2}}{2} \int_{0}^{L} \eta^{\prime \prime}(x)^{2} d x-\frac{1}{2\left(1+a_{1}\right)} \int_{0}^{L} l(x) \eta^{\prime}(x)^{2} d x+\frac{a_{1}}{8\left(1+a_{1}\right)} \int_{0}^{L} \eta^{\prime}(x)^{4} d x \\
\geq \frac{a_{2}}{2} \int_{0}^{L} \eta^{\prime \prime}(x)^{2} d x-\frac{1}{2\left(1+a_{1}\right)}\left[\int_{0}^{L} l(x)^{2} d x\right]^{\frac{1}{2}}\left[\int_{0}^{L} \eta^{\prime}(x)^{4} d x\right]^{\frac{1}{2}} \\
+\frac{a_{1}}{8\left(1+a_{1}\right)} \int_{0}^{L} \eta^{\prime}(x)^{4} d x .
\end{gathered}
$$

Setting

$$
K_{2}=\frac{1}{2\left(1+a_{1}\right)}\left[\int_{0}^{L} l(x)^{2} d x\right]^{\frac{1}{2}}
$$


and

$$
K_{3}=\frac{a_{1}}{8\left(1+a_{1}\right)},
$$

we have for $\eta \in V_{M}$

$$
M \geq \frac{a_{2}}{2}\left\|\eta^{\prime \prime}\right\|^{2}-K_{2}\left\|\eta^{\prime}\right\|_{L^{4}(0, L)}^{2}+K_{3}\left\|\eta^{\prime}\right\|_{L^{4}(0, L)}^{4} .
$$

With $\gamma=\min \left\{\frac{a_{2}}{2}, K_{3}\right\}$ there follows

$$
\begin{aligned}
M & \geq \gamma\left[\left\|\eta^{\prime \prime}\right\|^{2}+\left\|\eta^{\prime}\right\|_{L^{4}(0, L)}^{4}\right]-K_{2}\left[\left\|\eta^{\prime}\right\|_{L^{4}(0, L)}^{4}\right]^{\frac{1}{2}} \\
& \geq \gamma\left[\left\|\eta^{\prime \prime}\right\|^{2}+\left\|\eta^{\prime}\right\|_{L^{4}(0, L)}^{4}\right]-K_{2}\left[\left\|\eta^{\prime \prime}\right\|^{2}+\left\|\eta^{\prime}\right\|_{L^{4}(0, L)}^{4}\right]^{\frac{1}{2}}
\end{aligned}
$$

Then, with

$$
\delta=\left[\left\|\eta^{\prime \prime}\right\|^{2}+\left\|\eta^{\prime}\right\|_{L^{4}(0, L)}^{4}\right]^{\frac{1}{2}},
$$

we have

$$
M \geq \gamma \delta^{2}-K_{2} \delta
$$

i.e.,

$$
\gamma \delta^{2}-K_{2} \delta-M \leq 0
$$

from which it follows easily that

$$
0 \leq \delta \leq \frac{K_{2}+\left[K_{2}^{2}+4 \gamma M\right]^{\frac{1}{2}}}{2 \gamma} \equiv M_{1},
$$

and from (4.13),

$$
\left[\left\|\eta^{\prime \prime}\right\|^{2}+\left\|\eta^{\prime}\right\|_{L^{4}(0, L)}^{4}\right]^{\frac{1}{2}} \leq M_{1}
$$

implying $\left\|\eta^{\prime \prime}\right\| \leq M_{1}$, as we proposed to show.

Now suppose $\left\{\eta_{k}\right\}_{k=1}^{\infty}$ is a minimizing sequence for $\mathbf{J}(\eta)$ in $V_{M}$ :

$$
M \geq \mathbf{J}\left(\eta_{k}\right) \rightarrow d .
$$

Then we have $\left\|\eta_{k}^{\prime \prime}\right\| \leq M_{1}$, and there is a subsequence, which we continue to denote by $\left\{\eta_{k}\right\}_{k=1}^{\infty}$, converging weakly to a limit $\eta_{0}$ in $H^{2}(0, L)$, and thus converging strongly to the same limit in $H^{1}(0, L)$ and $W^{1,4}(0, L)$. From the formula (4.8) for $\mathbf{J}(\eta)$ and the limiting behavior

$$
\liminf \int_{0}^{L} \eta_{k}^{\prime \prime}(x)^{2} d x \geq \int_{0}^{L} \eta_{0}^{\prime \prime}(x)^{2} d x
$$

and

$$
\lim \int_{0}^{L} \eta_{k}^{\prime}(x)^{2(4)} d x=\int_{0}^{L} \eta_{0}^{\prime}(x)^{2(4)} d x
$$


it follows that

$$
d=\liminf \mathbf{J}\left(\eta_{k}\right)=\mathbf{J}\left(\eta_{0}\right) .
$$

We conclude that there is an element $\eta_{0} \in V_{2}$ such that $\mathbf{J}\left(\eta_{0}\right)=d$, and the proof of the theorem is complete.

The condition (4.9), which we may restate as

$$
\int_{0}^{L} x^{2} l(x) d x>L a_{2}\left(1+a_{1}\right)
$$

serves to characterize combinations of boundary and distributed longitudinal loads that produce nonzero equilibria. In the case of an endpoint load $g_{l}$, we have $l(x) \equiv-g_{l}$, and (4.14) is true just in case

$$
g_{l}<-\frac{3 a_{2}\left(1+a_{1}\right)}{L^{2}} .
$$

Clearly, when (4.14) is the case, $\eta(x) \equiv 0$ does not minimize the functional $\mathbf{J}(\eta)$. Since $\mathbf{J}(\eta)$, by Theorem 4.2 , has a minimizing $\eta_{0} \in V_{2}$, it follows that the boundary value problem (4.4)-(4.6) has $\eta_{0}$ as a nonzero solution. It is nonunique because $\mathbf{J}\left(\eta_{0}\right)=\mathbf{J}\left(-\eta_{0}\right)$. We note that no nonuniqueness occurs as long as $\mathbf{J}(\eta)$ remains strictly convex; in particular, this remains true as long as the quadratic part of $\mathbf{J}(\eta)$ is a positive definite quadratic form, i.e., as long as the operator

$$
\mathbf{L}(\eta)=a_{2} \eta^{\prime \prime \prime \prime}-\frac{g_{l}}{1+a_{1}} \eta^{\prime \prime},
$$

defined on the domain in $H^{4}(0, L)$ determined by boundary conditions (4.5) and (cf. (4.6))

$$
a_{2} \eta^{\prime \prime \prime}(L)-\frac{g_{l}}{1+a_{1}} \eta^{\prime}(L)=0,
$$

has only positive eigenvalues. For given positive values of $a_{1}$ and $a_{2}$, this will be true for (negative) values of $g_{l}$ in some interval $(-\gamma, 0)$; $-\gamma$ is the classical "buckling" load. For our nonlinear model, $\mathbf{J}(\eta)$ remains convex for $g_{l} \in[-\gamma, 0)$ because of the quartic term in (4.8); nonuniqueness sets in for $g_{l}<-\gamma$. For $g_{l} \in[-\gamma, 0)$, we have the "trivial" solution $\eta(x) \equiv 0$, but it should be noted that the complete state $\omega=(\xi, \eta) \neq(0,0)$ because (4.3) with $\eta(x) \equiv 0$ shows that

$$
\xi^{\prime}(x)=-\frac{g_{l}}{1+a_{1}},
$$

corresponding to a longitudinal compression varying linearly with respect to $x$.

In the case of a strictly distributed longitudinal load, i.e., $g_{l}=0$, it seems desirable to restate the condition (4.14) in terms of the load distribution function $g(x)$ :

$$
-3 L a_{2}\left(1+a_{1}\right)>\int_{0}^{L} 3 x^{2} \int_{x}^{L} g(s) d s d x=\int_{0}^{L} x^{3} g(x) d x .
$$

Under these circumstances, from our earlier remarks, there must be a nontrivial solution to (4.4)-(4.6). 
On the other hand, for all $\phi \in V_{2}$, the Gâteaux derivative of $\mathbf{J}(\eta)$ in the direction $\phi$ at a minimizing $\eta_{0}$ is

$$
D \mathbf{J}\left(\eta_{0}\right)(\phi)=\int_{0}^{L}\left[a_{2} \eta_{0}^{\prime \prime}(x) \phi^{\prime \prime}(x)-\frac{l(x)}{1+a_{1}} \eta_{0}^{\prime}(x) \phi^{\prime}(x)+\frac{a_{1} / 2}{1+a_{1}} \eta_{0}^{\prime}(x)^{3} \phi^{\prime}(x)\right] d x=0 .
$$

In the particular case when $\phi(x)=\eta_{0}$, we have

$$
D \mathbf{J}\left(\eta_{0}\right)\left(\eta_{0}\right)=\int_{0}^{L}\left[a_{2} \eta_{0}^{\prime \prime}(x)^{2}-\frac{l(x)}{1+a_{1}} \eta_{0}^{\prime}(x)^{2}+\frac{a_{1}}{2\left(1+a_{1}\right)} \eta_{0}^{\prime}(x)^{4}\right] d x=0 .
$$

With (cf. (4.10))

$$
\int_{0}^{L} \eta_{0}^{\prime \prime}(x)^{2} d x \geq \frac{1}{\gamma_{0}^{2}} \int_{0}^{L} \eta_{0}^{\prime}(x)^{2} d x
$$

we then must have

$$
0 \geq \int_{0}^{L}\left\{\left[\frac{a_{2}}{\gamma_{0}^{2}}-\frac{l(x)}{1+a_{1}}\right] \eta_{0}^{\prime}(x)^{2}+\frac{a_{1}}{2\left(1+a_{1}\right)} \eta_{0}^{\prime}(x)^{4}\right\} d x .
$$

If

$$
\frac{a_{2}}{\gamma_{0}^{2}} \geq \frac{l(x)}{1+a_{1}}, \quad x \in[0, L]
$$

then (4.16) implies

$$
0 \geq \int_{0}^{L}\left[\frac{a_{1}}{2\left(1+a_{1}\right)} \eta_{0}^{\prime}(x)^{4}\right] d x \geq 0
$$

and we see that $\eta_{0}=0$ is the only possibility.

5. Buckling in a noncantilevered beam. We have, for definiteness, carried out the preceding analyses in the so-called cantilever configuration of the beam. In that case, however, the nonzero equilibria which we have shown to exist for sufficiently large negatively directed longitudinal loads, when actually computed, may be seen to correspond to lateral displacement functions $\eta(x)$, which are monotone functions of $x$; when the longitudinal loading exceeds a certain level, the beam sways and "tips over" to a nonzero configuration. This is shown in Figure 6.2 since the numerical solution of the beam equations is discussed in section 6 . What is notable for this simple model, as compared with classical linear analysis, is the finite deformation constituting the new equilibrium. There are many models giving finite deformation solutions in such systems, going back to Euler, in which there are large purely transverse end loadings resulting in nonuniqueness of finite deformation solutions; see [12].

Classical buckling studies are usually concerned with situations where both ends are fixed. These include the case which is usually described as a "simply supported" beam, where we have

$$
\eta(0)=\eta(L)=0
$$

but do not require $\eta^{\prime}(0)=0$. In this case, the other boundary conditions applying in the case of a longitudinal load applied at $x=L$ (but no transverse loading there) are

$$
\xi(0)=0, \quad \eta^{\prime \prime}(0)=\eta^{\prime \prime}(L)=0, \quad\left(1+a_{1}\right) \xi^{\prime}(L)+\eta^{\prime}(L)^{2} / 2=g_{l} .
$$


One could also consider the case where the beam is clamped at $x=0$ and simply supported at $x=L$. In that case, with the same loading, we have

$$
\begin{gathered}
\eta(0)=\eta^{\prime}(0)=\eta(L)=\eta^{\prime \prime}(L)=0, \\
\xi(0)=0, \quad\left(1+a_{1}\right) \xi^{\prime}(L)+\eta^{\prime}(L)^{2} / 2=g_{l} .
\end{gathered}
$$

We will study the latter case, i.e., (5.1) and (5.2), for a simple longitudinal load $g_{l}$ applied at $x=L$. We begin by noting that, when $\eta(x) \equiv 0$, we have (cf. (4.8))

$$
\begin{aligned}
J(\xi, 0) & =\frac{1}{2} \int_{0}^{L}\left[a_{1} \xi^{\prime}(x)^{2}+\xi^{\prime}(x)^{2}\right] d x-g_{l} \xi(L) \\
& =\frac{1}{2} \int_{0}^{L}\left[\left(1+a_{1}\right) \xi^{\prime}(x)^{2}-2 g_{l} \xi^{\prime}(x)\right] d x \\
& =\frac{1+a_{1}}{2} \int_{0}^{L}\left\{\left[\xi^{\prime}(x)-\frac{g_{l}}{1+a_{1}}\right]^{2}+\left[\frac{g_{l}}{1+a_{1}}\right]^{2}\right\} d x
\end{aligned}
$$

showing that the minimum occurs for

$$
\xi^{\prime}(x) \equiv \frac{g_{l}}{1+a_{1}}, \text { i.e., } \xi(x) \equiv \frac{g_{l} x}{1+a_{1}} .
$$

This state represents the "trivial" equilibrium; it can be shown to be the only equilibrium if $g_{l}$ is nonnegative or negative and small. To construct, for sufficiently large negative $g_{l}$, a state $\left(\xi_{0}, \eta_{0}\right) \in \mathcal{V}$ for which $J\left(\xi_{0}, \eta_{0}\right)$ is less than $J\left(\frac{x g_{l}}{1+a_{1}}, 0\right)$, we begin by rewriting $J(\xi, \eta)$ in the form

$$
\begin{aligned}
J(\xi, \eta) & =\frac{1+a_{1}}{2} \int_{0}^{L}\left\{\left[\xi^{\prime}(x)-\frac{g_{l}}{1+a_{1}}\right]^{2}+\left(\frac{g_{l}}{1+a_{1}}\right)^{2}\right\} d x \\
& +\frac{1}{2} \int_{0}^{L}\left\{a_{2} \eta^{\prime \prime}(x)^{2}+\xi^{\prime}(x) \eta^{\prime}(x)^{2}+\frac{1}{4} \eta^{\prime}(x)^{4}\right\} d x .
\end{aligned}
$$

Taking $\xi(x)$ as in (5.3), we have

$$
\begin{aligned}
& J\left(\frac{x g_{l}}{1+a_{1}}, \eta\right)=\frac{1}{2} \frac{L g_{l}^{2}}{1+a_{1}} \\
& \quad+\frac{1}{2} \int_{0}^{L}\left\{a_{2} \eta^{\prime \prime}(x)^{2}+\frac{g_{l}}{1+a_{1}} \eta^{\prime}(x)^{2}+\frac{1}{4} \eta^{\prime}(x)^{4}\right\} d x
\end{aligned}
$$

the first term here being $J\left(\frac{x g_{l}}{1+a_{1}}, 0\right)$. We satisfy the boundary conditions with any of the choices

$$
\eta_{\alpha}(x)=\alpha\left(x^{2}-x^{3} / L\right), \quad \alpha \text { real. }
$$

If (5.5) is substituted for $\eta(x)$ in (5.4), we see that $J$ has the form

$$
J\left(\frac{x g_{l}}{1+a_{1}}, \eta_{\alpha}\right)=\frac{L g_{l}^{2}}{2\left(1+a_{1}\right)}+p \alpha^{2}+q \alpha^{4}
$$


where calculation shows that

$$
p=2 a_{2} L+\frac{11 g_{l}}{3\left(1+a_{1}\right)} L^{3} .
$$

We will have

$$
J\left(\frac{x g_{l}}{1+a_{1}}, \eta_{a}\right)<J\left(\frac{x g_{l}}{1+a_{1}}, 0\right)
$$

for sufficiently smalll nonzero $\alpha$ if $p$ is negative, i.e., if

$$
g_{l}<-\frac{6}{11 L^{2}} a_{2}\left(1+a_{1}\right)
$$

Most of the conclusions drawn in the previous section for the cantilever case can be obtained, with appropriate modifications, in this case as well; we will not pursue these results formally in the interests of brevity.

6. Numerical experiments. In this section, we present calculations to obtain approximations of nontrivial solutions of the boundary value problems (2.19)-(2.23). Toward this end, we consider the differential equation with $f=0$ given by

$$
a_{2} \eta^{\prime \prime \prime \prime}+\left(\frac{l}{1+a_{1}} \eta^{\prime}\right)^{\prime}-\left(\frac{a_{1}}{2\left(1+a_{1}\right)}\left(\eta^{\prime}\right)^{3}\right)^{\prime}=0 \text { in }(0, L)
$$

and essential boundary conditions at $x=0$

$$
\xi(0)=\eta(0)=\eta^{\prime}(0)=0
$$

along with the free boundary conditions at $x=L$

$$
\begin{gathered}
\eta^{\prime}(L)=0 \\
{\left[\left(\xi^{\prime}+\frac{\left(\eta^{\prime}\right)^{2}}{2}\right) \eta^{\prime}-a_{2} \eta^{\prime \prime \prime}\right](L)=0}
\end{gathered}
$$

or with pinned boundary conditions at $x=L$

$$
\eta(L)=\eta^{\prime \prime}(L)=0
$$

that have been discussed in previous sections. Define the Hilbert spaces

$$
V=\left\{\phi \in H^{2}(0, L): \eta(0)=\eta^{\prime}(0)=0\right\}
$$

and

$$
V_{0}=\{\phi \in V: \eta(L)=0\}
$$

and the functional

$$
j(\eta)=\int_{0}^{L}\left\{\frac{a_{2}}{2} \eta^{\prime \prime}(x)^{2}-\frac{l(x)}{2\left(1+a_{1}\right)} \eta^{\prime}(x)^{2}+\frac{a_{1}}{8\left(1+a_{1}\right)} \eta^{\prime}(x)^{4}\right\} d x .
$$

The longitudinal force at $x=L$ has been included in $l$. In fact, we take $l=g_{l}$. 


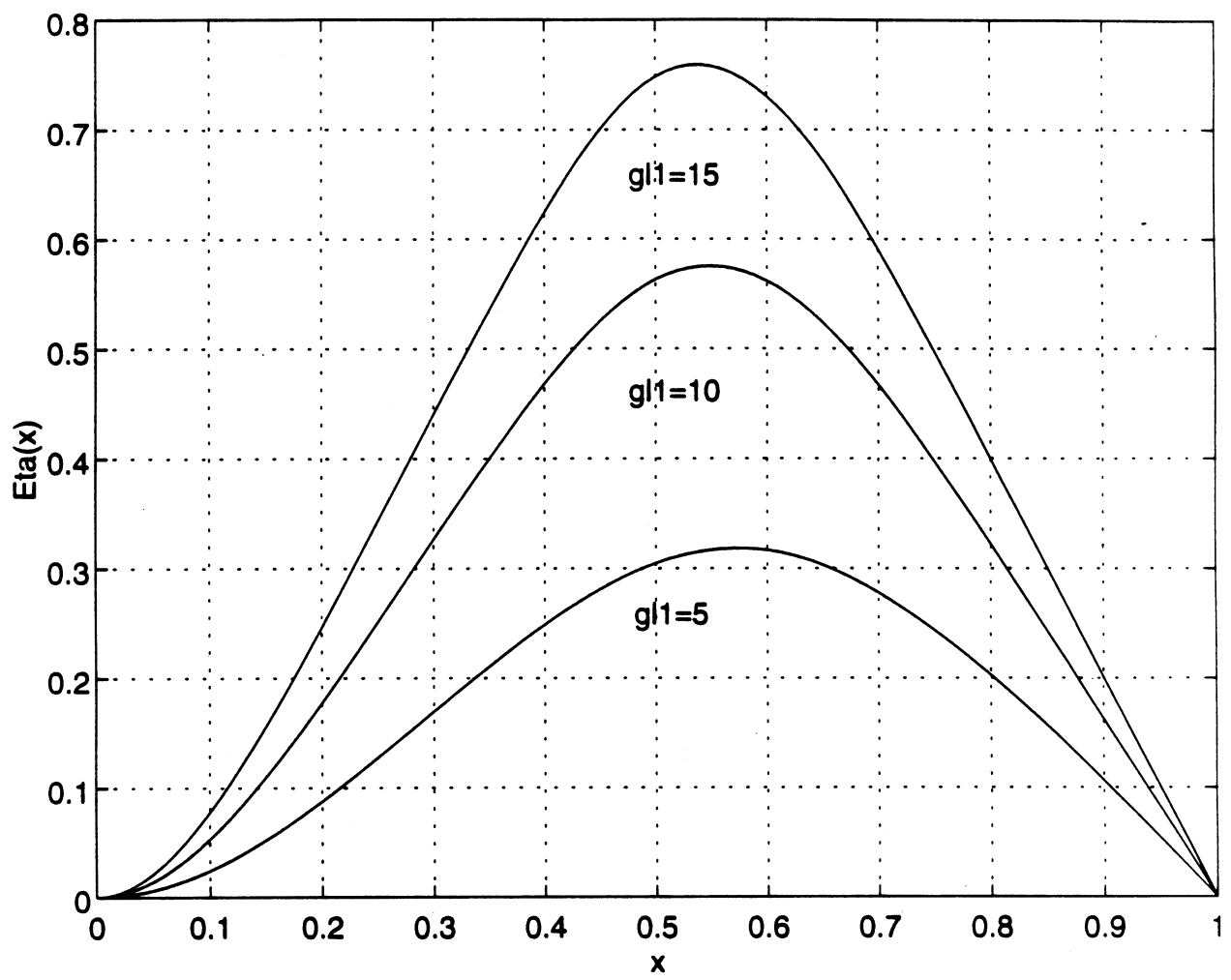

FIG. 6.1. Eta for beam pinned at $x=L$ for different values of gl1.

We consider the minimization of the functional j over subspaces of $V$ and $V_{0}$ to obtain buckling deformations. Let $L=1$, and let $(0,1)$ be divided into $N$ subintervals of equal length. Let $\left\{B_{k}\right\}_{k=1}^{M}$ be cubic B-splines defined with respect to the partition and such that they satisfy the boundary conditions at $x=0$. It is also convenient for the free case to include the condition that $B_{k}^{\prime \prime}(1)=0$ as well. Of course, in the pinned case, $B_{k}(1)=0$ is imposed as an essential boundary condition. It follows that in both cases the number of basis elements $M$ equals $N$; cf. [15]. Let $V^{N}$ and $V_{0}^{N}=$ $\operatorname{span}\left\{B_{k}\right\}_{k=1}^{M}$ in the appropriate cases. Define the $M \times M$ matrices

$$
\left(G_{2}\right)_{i j}=a_{2} \int_{0}^{1} B_{i}^{\prime \prime}(x) B_{j}^{\prime \prime}(x) d x
$$

and

$$
\left(G_{1}\right)_{i j}=\frac{1}{\left(1+a_{1}\right)} \int_{0}^{1} B_{i}^{\prime}(x) B_{j}^{\prime}(x) d x
$$

and the functional on $\mathbf{R}^{M}$

$$
F(c)=\frac{a_{1}}{2\left(1+a_{1}\right)} \int_{0}^{1}\left(\sum_{i=1}^{M} c_{i} B_{i}(x)\right)^{4} d x,
$$

where $c=\operatorname{col}\left(c_{1}, \ldots, c_{M}\right)$. The functional $j(\cdot)$ is given as

$$
j(c)=c^{*}\left(G_{2}-g_{l} G_{1}\right) c+F(c) .
$$




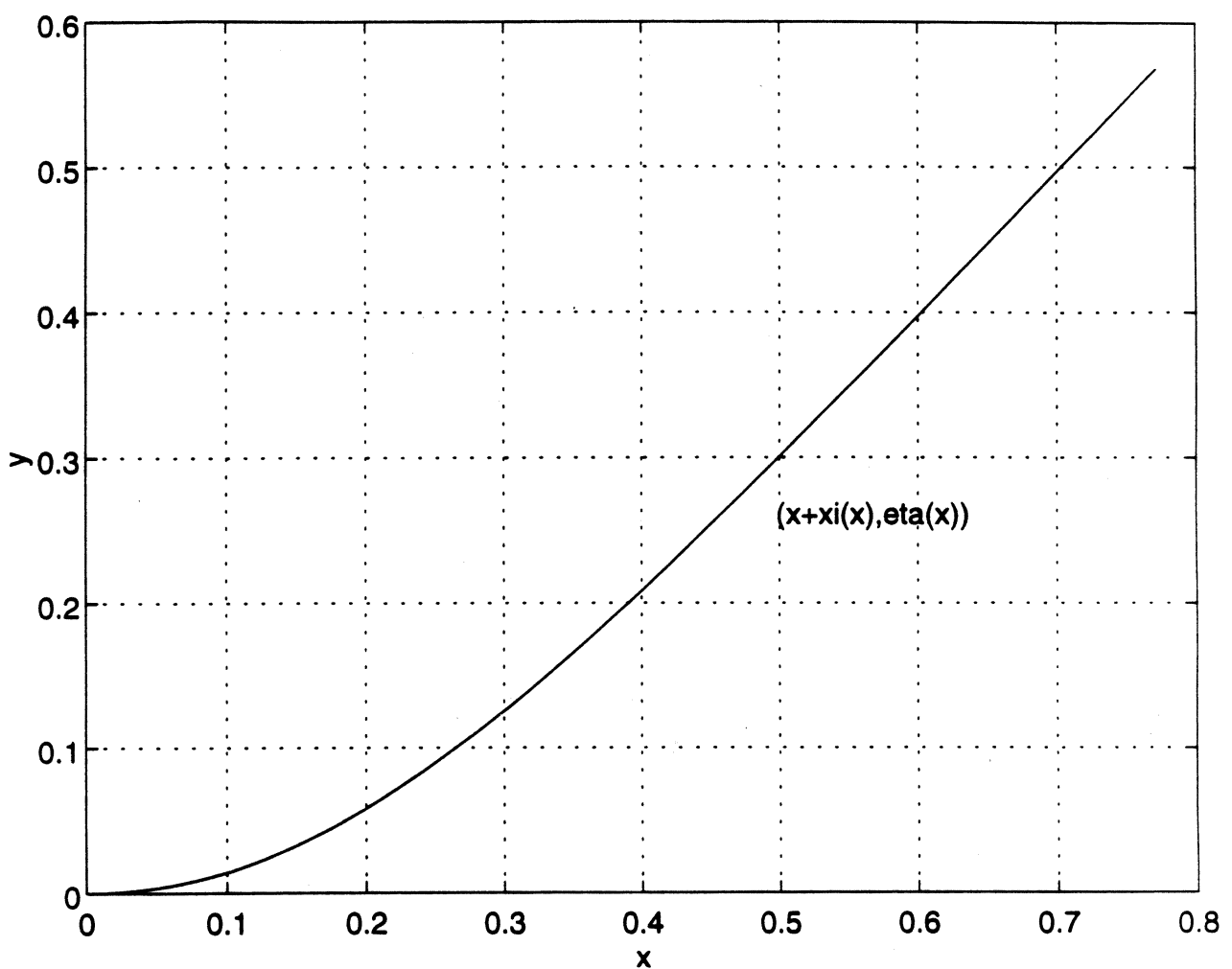

FIG. 6.2. Cantilevered beam displacement for gl1 $=2$.

Since the partial derivative of $F(c)$ with respect to $c_{k}$ is given by

$$
D_{k} F(c)=\frac{2 a_{1}}{1+a_{1}} \int_{0}^{1}\left(\sum_{i=1}^{M} c_{i} B_{i}(x)\right)^{3} B_{k}(x) d x,
$$

the corresponding partial derivative of $j(\cdot)$ is given by

$$
D_{k} j(c)=\left[\left(G_{2}-g_{l} G_{1}\right]_{k}+D_{k} F(c)\right.
$$

for $k=1, \ldots, M$. A steepest descent method is used to minimize $j(c)$ over $\mathbf{R}^{M}$.

Setting the beam thickness $=0.1, \mu=0.25$, and taking $N=8$, we choose $g_{l}$ such that $G_{2}-g_{l} G_{1}$ has a negative eigenvalue. If

$$
g_{l}>\pi^{2} a_{2}\left(1+a_{1}\right),
$$

then it is possible for there to be negative eigenvalues. Thus we set

$$
g_{l}=g_{l_{1}} \pi^{2} a_{2}\left(1+a_{1}\right) .
$$

The steepest descent iterations are initialized by an eigenvector associated with the negative eigenvalue. Figure 6.1 shows, for the purpose of comparison, the graphs of $\eta$ for the case in which the boundary is pinned at $x=L$ for various values of the longitudinal force factor $g_{l_{1}}$. Figure 6.2 portrays the finite element approximation of 
the beam displacement $x+\xi(x)$ versus $\eta(x)$ for a beam in a cantilevered configuration as discussed in section 5 in the case when $g_{l_{1}}=2$. The value $g_{l_{1}}=2$ is chosen for the aesthetic appearance of the graph. To obtain this result, it is necessary to approximate the solution of the boundary value problem (2.12), (2.19), and (2.22) for $\xi$ after the approximation for $\eta$ has been obtained. Piecewise linear elements on the same mesh as is used in solving for $\eta$ are used for this approximation.

\section{REFERENCES}

[1] R. A. Adams, Sobolev Spaces, Academic Press, New York, 1975.

[2] S. S. Antman, The Theory of Rods, Handbuch der Physik, Vol. VIa/2, Springer-Verlag, Berlin, 1972.

[3] S. S. Antman, Ordinary differential equations of nonlinear elasticity I: Foundations of the theories of nonlinearly elastic rods and shells, Arch. Ration. Mech. Anal., 61 (1976), pp. 307-351.

[4] S. S. Antman, Ordinary differential equations of nonlinear elasticity II: Existence and regularity theory for conservative boundary value problems, Arch. Ration. Mech. Anal., 61 (1976), pp. 353-393.

[5] S. S. Antman, Nonlinear Problems of Elasticity, Springer-Verlag, New York, 1995.

[6] P. C. Chou and N. J. Pagano, Elasticity: Tensor, Dyadic and Engineering Approaches, D. Van Nostrand, Princeton, NJ, 1967, Dover, New York, 1992.

[7] D. Y. Gao and G. Strang, Geometric nonlinearity, potential energy, complementary energy, and the gap function, Quart. Appl. Math., 47 (1989), pp. 487-504.

[8] D. Y. Gao and D. L. Russell, An extended beam theory for smart materials applications I: Extended beam models, duality theory, and finite element simulations, Appl. Math. Optim., 34 (1996), pp. 279-298.

[9] S. C. Hunter, Mechanics of Continuous Media, 2nd ed., Halstead, New York, 1983.

[10] J. LAGNESE, Recent progress in exact boundary controllability and uniform stabilization of thin beams and plates, in Distributed Parameter Control Systmes, G. Chen, E. B. Lee, W. Littman, and L. Markus, eds., Marcel Dekker, New York, 1991, pp. 61-111.

[11] J. Lagnese And J.-L. Lions, Modelling, Analysis, and Control of Thin Plates, Springer-Verlag, New York, 1989.

[12] A. E. H. Love, Mathematical Theory of Elasticity, 4th ed., Dover, New York, 1944.

[13] E. P. Popov, Nonlinear Problems in the Statics of Thin Rods, Gostekhteorizdat, Leningrad, 1948.

[14] D. L. Russell, A static formation theory for active elastic materials, in Proceedings of the IFIP Conference on Control of Distributed Parameter Systems (Laredo, Spain, 1994), Marcel Dekker, New York, 1995.

[15] M. Schultz, Spline Analysis, Prentice-Hall, Englewood Cliffs, NJ, 1973. 